\title{
IdeAs
}

Idées d'Amériques

$17 \mid 2021$

Villes et culture dans les Amériques

\section{«L'occasion unique de faire une bonne action » : la campagne du «Paris Tribune » en faveur de l'American Library in Paris}

\section{Nissa Ren Cannon}

Traducteur : Anne Reynès-Delobel

\section{(2) OpenEdition}

\section{Journals}

Édition électronique

URL : https://journals.openedition.org/ideas/11153

DOI : 10.4000/ideas. 11153

ISSN : 1950-5701

\section{Éditeur}

Institut des Amériques

\section{Référence électronique}

Nissa Ren Cannon, « «L'occasion unique de faire une bonne action » : la campagne du « Paris Tribune » en faveur de l'American Library in Paris », IdeAs [En ligne], 17 | 2021, mis en ligne le 01 mars 2021, consulté le 04 juin 2021. URL : http://journals.openedition.org/ideas/11153 ; DOl : https:// doi.org/10.4000/ideas.11153

Ce document a été généré automatiquement le 4 juin 2021.

\section{cc) (†)}

IdeAs - Idées d'Amériques est mis à disposition selon les termes de la licence Creative Commons Attribution - Pas d'Utilisation Commerciale - Pas de Modification 4.0 International. 


\title{
«L'occasion unique de faire une bonne action $»$ : la campagne du «Paris Tribune » en faveur de l'American Library in Paris
}

\author{
Nissa Ren Cannon \\ Traduction : Anne Reynès-Delobel
}

"Voici l'occasion unique pour les Américains de Paris de faire une bonne action", déclare l'édition parisienne du Chicago Tribune dans ses colonnes du 21 octobre 1919. Dans cet article, le journal encourage vivement ses lecteurs à soutenir financièrement la création de l'American Library in Paris. Le lendemain, le quotidien poursuit dans une veine sentimentale en demandant à ses lecteurs « lequel d'entre eux n'a jamais ressenti le besoin nostalgique de consulter des livres ou des revues de son pays natal, et de naviguer parmi les rayonnages, tout en bénéficiant de l'aide et des conseils avisés d'un bibliothécaire qualifié ?». Cette campagne menée par le bureau parisien du Tribune (officiellement titré Chicago Tribune European Edition et familièrement surnommé «Paris Tribune ») vise à maintenir une institution culturelle installée dans la capitale française depuis 1918, la salle de lecture du Library War Service (LWS), lequel était placé sous l'égide de l'American Library Association (ALA). Or, au moment où le Tribune fait paraitre son plaidoyer, la guerre est finie, la démobilisation est en cours et le programme mis place par l'ALA pour les troupes américaines est en passe de se terminer. Cette levée de fonds répond, de fait, à la demande des civils américains à Paris, dont le nombre ne cesse de croître et qui voient dans cette institution, selon les termes employés dans les pages du Tribune, "un lieu qui concentre nos intérêts intellectuels communs et notre culture spécifique ». 


\section{Une histoire partagée}

2 Le Paris Tribune et l'American Library ont une origine commune : tous deux ont été fondés dans le cadre du déploiement du corps expéditionnaire américain (American Expeditionary Forces ou AEF) en Europe durant Première Guerre mondiale et tous deux se sont réinventés après la démobilisation. Le 4 juillet 1917, en réponse à l'appel lancé par le ministère de la Guerre en direction de la presse afin de «tenir les troupes américaines succinctement informées de l'actualité nationale et, autant que faire se peut, de la situation en France ", le Chicago Tribune fait paraître le premier numéro de son « Army Edition ».

3 Cette "Army Edition », qui dispose de sa propre équipe de rédaction, présente son contenu en ces termes: "les meilleurs articles du Tribune, ainsi que des dessins humoristiques, une rubrique sportive, l'essentiel de l'actualité et une sélection de dépêches officielles ou semi-officielles revêtant un intérêt et une valeur politique ou militaire pour nos troupes engagées dans le conflit» (18 septembre 1917). Elle est publiée jusqu'au 18 février 1919, date à laquelle elle change son nom en «Paris Edition », puis peu de temps après, en "European Edition $»^{1}$ de sorte à répondre à l'évolution de la communauté américaine à Paris, dans l'immédiate après-guerre.

4 En pratique, le passage d'un lectorat militaire à un lectorat civil se fait toutefois de manière graduelle, comme le laissent observer les réclames publiées tout au long de l'année 1919 dans les pages du quotidien pour le compte du grand magasin Au Bon Marché. Au début de l'année, ces réclames font l'éloge de "vêtements et équipements militaires » destinés à la clientèle masculine des soldats. Quelques mois plus tard, en juillet, dans le "Paris Edition » c'est la même clientèle qui est ciblée, mais avec un produit différent : cette fois, il s'agit de « gants, dentelles et bas de soie » que les soldats américains pourront emporter dans leurs bagages comme autant de «souvenirs de Paris » pour en faire présent à leurs proches. Néanmoins, ce n'est qu'en fin d'année que le changement de lectorat peut s'observer de manière manifeste, comme en témoigne cette réclame, parue dans le numéro de septembre, qui vante les «robes de saison, blouses et corsages ", ainsi que les très sélect salon de thé et restaurant de l'enseigne et qui, à l'évidence, vise désormais une clientèle féminine. Ces changements reflètent la transformation de la population américaine à Paris après-guerre: aux soldats succèdent les hommes d'affaires et leurs familles, les journalistes, les étudiants et les artistes ${ }^{2}$.

5 Cette évolution démographique a un impact non négligeable sur la salle de lecture de l'ALA à Paris. En 1917, l'ALA gère le LWS qui a pour mission de fournir des livres aux soldats. Rapidement, cette initiative s'étend à travers les États-Unis et l'Europe ${ }^{3}$. Au pic de son activité, l'ALA gère 32 bibliothèques abritées dans des bâtiments, 35 grandes bibliothèques de camp et 237 bibliothèques dans des hôpitaux et des établissements de la Croix-Rouge. En Europe, on trouve des bibliothèques de prêt :

[dans les] trains sanitaires, les sections de l'American Ambulance, les Foyers du soldat de l'Union franco-américaine, les clubs pour infirmières et les hostess houses, aux bases navales et à bord des bâtiments de la flotte des États-Unis, dans les centres récréatifs, comme le Soldiers' and Sailors' Club, les locaux de l'organisation Moose, l'University Union, les foyers de la YMCA, les locaux de l'Armée du Salut et des Knights of Columbus, et les hôpitaux de la Croix-Rouge. (« New Library Opened », 30 août 1918) 
Illustration 1 : Soldats dans l'une des salles de lectures du Library War Service gérées par l'ALA.

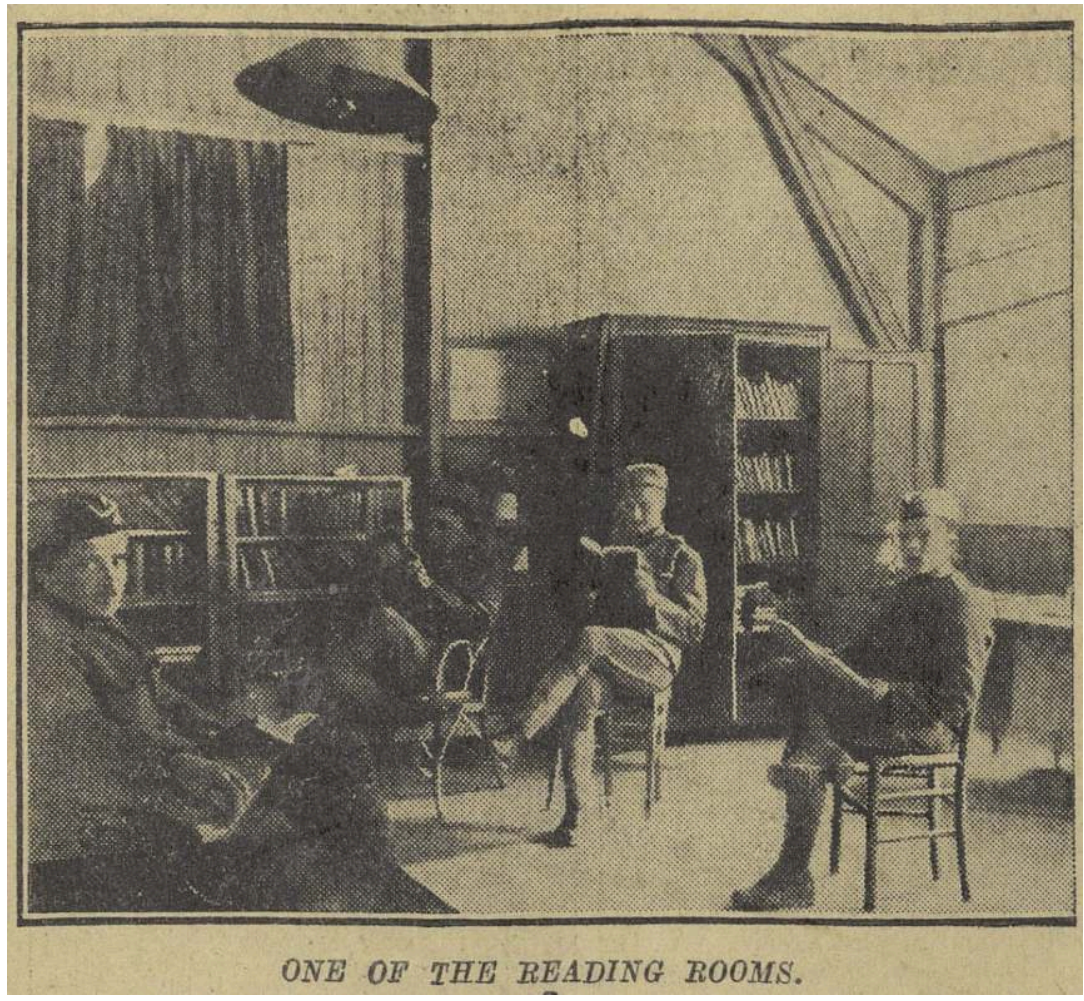

New York Herald Paris Edition, 20 octobre, 1919, p. 4.

6 À partir de 1918, le LWS prend ses quartiers au 10, rue de l'Elysée, à proximité de la place de la Concorde, dans une élégante demeure autrefois occupée par la nonciature apostolique à Paris. Lorsque débute la lente démobilisation des armées alliées, un nombre croissant de soldats et de civils américains converge vers la capitale. La bibliothèque américaine s'ouvre alors au public et, comme le souligne Mary Niles Maack, "cette foule des plus composites ne tarde pas à faire usage de ses services " (Maack M., 2005 : 401). La 2 octobre 1919, son directeur, Burton Stevenson, observe «qu'en dépit du rapatriement des troupes, le volume des prêts n'a jamais été aussi important. Un nombre sans cesse croissant de civils américains et britanniques recourt aux services de la bibliothèque pour obtenir des livres et des revues ».

7 Les origines du Tribune et celle de l'American Library se confondent. Trois semaines après son lancement, dans son "Army Edition ", le Tribune informe ses lecteurs que l'ALA «a été mandatée par la Commission sur les activités des camps d'entraînement $\mathrm{du}$ ministère de la Guerre pour offrir des services de bibliothèque dans les 32 cantonnements et les camps d'entraînement des divisions de la garde nationale » (26 juillet 1917). Le quotidien se fait régulièrement l'écho des avancées de cette entreprise. Ainsi, en janvier 1918, il annonce que le « War Service Committee de l'American Library Association a déjà acheminé plus d'un demi-million d'ouvrages vers les camps d'entraînement (29 janvier 1919).

8 Tandis que les opérations se poursuivent, le journal rapporte que «110 caisses contenant des livres (romans, usuels, dictionnaires, livres d'histoire et ouvrages scientifiques) rangés dans des étagères, comme dans une bibliothèque américaine moderne" étaient arrivées en France (2 mai 1918). Toutefois la nouvelle la plus importante pour les soldats américains à Paris est celle, le 30 août 1918, de l'ouverture 
"d'une nouvelle bibliothèque dans la capitale à destination des soldats et des autres membres du personnel du corps expéditionnaire américain ", dotée d'une salle de lecture proposant les derniers titres de la presse américaine et d'un service de référence.

\section{Une bibliothèque en crise}

9 Le Tribune ne se contente pas d'informer ses lecteurs des activités de l'American Library, mais fait également fonction de canal de communication entre les ressortissants américains installés à Paris et l'institution. Ainsi, le 20 mai 1919, il fait paraître un article qui incite les "Yanks" à "écrire à l'ALA s'ils estiment que l'approvisionnement en livres est insuffisant ». La date de cet article pourrait donner à penser qu'en 1919, les opérations du LWS sont en phase ascendante, mais elles ont, en réalité, déjà atteint la phase de clôture, et la question qui se pose désormais concerne l'avenir du LWS. Le "Paris Herald», principal concurrent du Tribune en France, rapporte le 24 juillet que la bibliothèque « ne disposant ni des fonds ni des compétences pour conduire le projet une fois la démobilisation achevée », « il est urgent de lever des fonds si l'on entend œuvrer pour son maintien ». Courant juillet, les deux quotidiens informent leurs lecteurs des projets élaborés pour préserver les livres qui sont désormais acheminés depuis les camps d'entraînement vers un entrepôt parisien où ils seront "triés et distribués à diverses institutions». On estime que sur "les deux millions et demi de livres expédiés en France, plus d'un million ont été égarés ou sont hors d'usage. En moyenne, plus de cinq cent mille ouvrages sont en cours de réacheminement vers les États-Unis ou seront expédiés prochainement » (31 juillet 1919).

Illustration 2 : Dans l'une salle du courrier de l'ALA, des femmes préparent un envoi de livres à destination du LWS.

\section{The American Library Association Is Not Demobilizing}

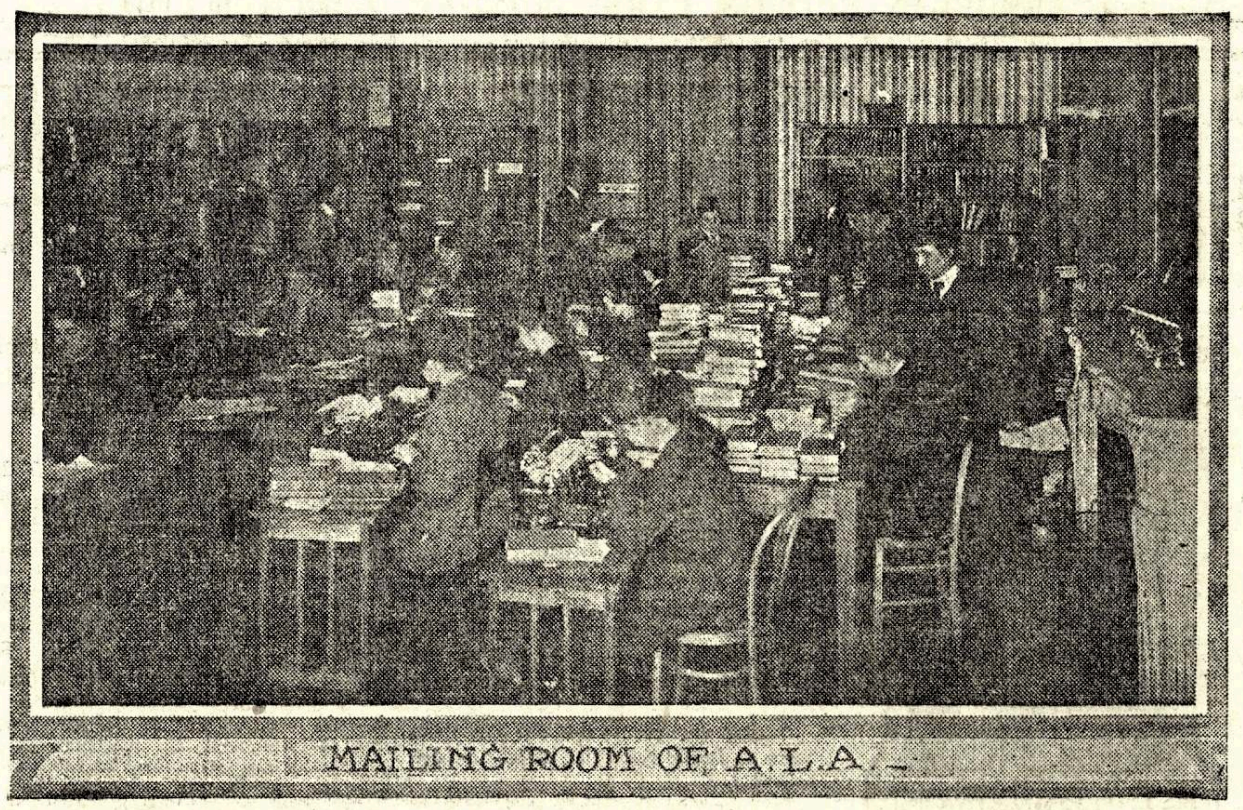

New York Herald Paris Edition, 10 mars 1919, p. 2. 
Néanmoins, nul ne souhaite vraiment la dispersion des ouvrages ni la fermeture de la salle de lecture, très fréquentée, du 10 , rue de l'élysée. Si le directeur de la bibliothèque, Burt Stevenson, pense pour sa part que « l'ALA n'est tenue en rien de faire cadeau d'une bibliothèque aux [civils] américains ayant choisi d'élire résidence à Paris " et que " cette communauté devrait prendre ce service à sa charge, comme il est d'usage " (Maak M., 2005 : 401), son projet n'est pas de fermer la bibliothèque, mais de trouver sur place les fonds nécessaires à son maintien. À ses yeux, il est important de « laisser une collection permanente qui pourra constituer le fonds d'une bibliothèque publique » en vue de "faciliter l'accès des Américains en France et du grand public à des informations faisant autorité sur les États-Unis»et de «contribuer au perfectionnement des relations franco-américaines » («Public Library in Paris »). À l'automne 1919, une campagne de sauvegarde de la bibliothèque est ainsi lancée et promue par les périodiques qui couvrent depuis longtemps toutes les opérations du LWS.

\section{La première campagne}

11 Le 20 octobre 1919, le «Paris Herald » informe ses lecteurs que « le maintien à Paris de l'American Library, érigée en l'honneur de l'esprit d'entreprise et de la littérature des États-Unis, exige la collecte de cent cinquante mille francs dans les deux prochaines semaines ». La rédaction du Tribune incite ses lecteurs à lui faire parvenir sans tarder un "chèque de cinq, dix, cent ou mille francs", charge à elle de collecter les dons et d'informer ses lecteurs chaque jour du montant obtenu qui sera intégralement remis à l'American Library (21 octobre 1919). La mobilisation des souscripteurs américains de Paris est telle qu'en octobre et novembre, il ne se passe pas un jour sans qu'il soit fait mention de la collecte (ainsi que de l'identité des donateurs) dans les colonnes, voire à la une, du Tribune et du Herald.

Pour convaincre les plus réticents, le Tribune n'hésite pas à jouer sur la corde sensible. Il décrit la bibliothèque comme un lieu "qu'il faut préserver à tout prix, ne serait-ce qu'en souvenir de ces jeunes et valeureux soldats pour qui elle fut un refuge et un havre de paix, loin des terreurs et des atrocités des combats ", et qui trouvera sa place dans les annales de la nation en tant «qu'agent de liaison entre ceux qui sont restés dans notre grand pays et ceux que la fortune a conduit à s'installer pour un temps dans cette capitale cosmopolite». Le journal faire encore appel au patriotisme de ses lecteurs en affirmant que la bibliothèque « est le maillon le plus solide de la chaîne qui relie les Américains de Paris à leur pays natal en perpétuelle mutation » (22 octobre 1919).

13 La campagne de sauvetage de l'American Library, relayée par le Herald et le Tribune, réalise son objectif au-delà de toute attente : la somme collectée est, de fait, deux fois supérieure à celle que Stevenson avait estimée nécessaire pour assurer la solvabilité de la bibliothèque jusque fin $1920^{4}$. En mai 1920, l'ALP se constitue en société privée, avec trois objectifs : "servir de mémorial aux soldats américains pour lesquels elle a été créée ", promouvoir " la connaissance de la littérature, des institutions et de la pensée américaines en France » et " compléter les maigres fonds d'ouvrages américains dans les bibliothèques publiques parisiennes existantes » («Some Facts »). L'ALA se retire du projet en exprimant le souhait que la bibliothèque «continue d'exister en tant que 
bibliothèque américaine et ainsi de témoigner de manière adéquate du mode de vie et de la façon de penser des Américains » (cité in Thompson S., 1964 : 180).

\section{Conclusion}

14 L'issue positive des campagnes menées par les rédactions des quotidiens américains à Paris pour conférer une pérennité institutionnelle à l'ALP marque le début d'une longue collaboration qui évoluera au fil des ans, à l'instar de la communauté américaine à Paris. Au début des années 1920, les éditions parisiennes du Tribune et du Herald publient avec le concours de la bibliothèque des rubriques littéraires hebdomadaires comprenant des nouvelles et des recensions. Pendant des dizaines d'années, les deux quotidiens continuent à lancer des souscriptions et à recevoir des dons pour le compte de la bibliothèque. Et si May Birkhead peut affirmer dans sa chronique mondaine, à la une du Tribune, que « les touristes passent mais l'American Library in Paris est là pour durer " (Birkhead M., 1927), c'est en grande partie grâce aux efforts déployés par ces journaux.

\section{BIBLIOGRAPHIE}

Birkhead, May, “Today in Society,” The Chicago Tribune, Paris Edition, April 3 1927, p. 1.

Ex Libris, "Some Facts about the American Library in Paris," Ex Libris, vol. 1, n 7, 1924, p. 201.

Green, Nancy L., The Other Americans in Paris: Businessmen, Countesses, Wayward Youth, 1880-1941, Chicago, The University of Chicago Press, 2014.

Maack, Mary Niles, “American Bookwomen in Paris during the 1920s," Libraries \& Culture, vol. 40, $\mathrm{n}^{\mathrm{o}} 3,2005$, p. 399-415.

Stauffer, Suzanne M., "Let Us Forget this Cherishing of Women in Library Work: Women in the American Library War Service, 1918-1920," Libraries: Culture, History, and Society, vol. 3, n 2, 2019, p. 155-174.

Thompson, Susan Otis, "The American Library in Paris: An International Development in the American Library Movement," The Library Quarterly: Information, Community, Policy, vol. 34, $\mathrm{n}^{\circ} 2$, 1964, p. 179-90.

Weber, Ronald. News of Paris: American Journalists in The City of Light Between the Wars, Chicago, Ivan R. Dee Press, 2006.

\section{NOTES}

1. Dans son ouvrage, cité en bibliographie, Ronald Weber retrace brièvement la genèse de «l'Army Edition » du Tribune. 
2. Voir dans la bibliographie, l'ouvrage que Nancy Green a consacré à la colonie américaine à Paris, pendant l'entre-deux-guerres.

3. Pour une présentation plus détaillée de ce programme, voir Stauffer, S., 2019.

4. L'ouvrage de $S$. Thompson retrace l'histoire de l'ALP de manière très documentée.

\section{AUTEURS}

\section{NISSA REN CANNON}

Nissa Ren Cannon est maîtresse de conférences à l'Université de Stanford (Programme Écriture et Rhétorique). Ses recherches portent sur le modernisme transatlantique, la question de la citoyenneté et la culture de l'imprimé. Elle a publié des articles dans symplokë et Journal of Modern Periodical Studies. D'autres articles sont à paraître dans ELN et Modernism/modernity. Elle a été postdoctoral Fellow, au Kilachand Honors College, de l'Université de Boston. nissaren@gmail.com 